\title{
Kondo Breakdown as a Selective Mott Transition in the Anderson Lattice
}

\author{
C. Pépin \\ SPhT, CEA-Saclay, L'Orme des Merisiers, \\ 91191 Gif-sur-Yvette, France
}

(Dated: October 10, 2018)

\begin{abstract}
We show within the slave boson technique, that the Anderson lattice model exhibits a Kondo breakdown quantum critical point (KB-QCP) where the hybridization goes to zero at zero temperature. At this fixed point, the f-electrons experience as well a selective Mott transition separating a local-moment phase from a Kondo-screened phase. The presence of a multi-scale QCP in the Anderson lattice in the absence of magnetism is discussed in the context of heavy fermion compounds. This study is the first evidence for a selective Mott transition in the Anderson lattice.

PACS numbers: 71.27.+a, 72.15.Qm, 75.20.Hr, 75.30.Mb
\end{abstract}

Quantum criticality in heavy-electron materials has attracted a substantial interest recently, mainly triggered by the remarkable metallic properties of these compounds [1]. The observation of anomalous exponents in both transport (exponents for the temperature dependence of the resistivity less than two) and thermodynamics ( the specific heat coefficient doesn't saturate at low temperatures) contradicts the universal predictions of the Landau Fermi liquid theories of metals [2, 3]. It has been suggested early that deviations from the Landau theory of metals can be explained by the proximity to a zero temperature phase transition, or QCP. Close to such a fixed point, the interactions with quantum critical massless modes substantially shorten the conduction electron lifetime at the Fermi surface, affecting the observable properties of the metal. Since heavy fermion compounds are strongly metallic and magnetic materials, QCPs towards itinerant antiferromagnetism (AF) have been first studied [4, [5, [6, 7]. Although they destabilize the Fermi liquid, quantum fluctuations in that case are not strong enough to explain both quasi-linear resistivity and anomalous thermodynamics properties in 3D. A new class of theories have then emerged [2, 8, 9]. Relying on Doniach's [11] observation that the Kondo effect and the anti-ferromagnetism are in competition in the Kondo lattice, these authors suggested that, at the magnetic QCP, another energy scale vanishes called "effective Kondo temperature". This new scale signals the breakdown of the heavy electron metal. Recently, this scenario of two energy scales vanishing congruently at the same point in the phase diagram has been challenged in another direction. A candidate for the Kondo breakdown quantum critical point (KB-QCP) has been found in the Kondo-Heisenberg lattice model [10, 12, 13] as a fixed point for which the hybridization between impurity electrons and conduction electrons vanishes. This new fixed point is intrinsically multi-scale[13]. Two distinct regimes are distinguished. Below a small energy threshold $E^{*}$ which depends on the $\mathrm{f}$ - and c- electron band structure (the typical value of $E^{*}$ ranges from $1 \mathrm{mK}$ to
$100 \mathrm{mK}$ ) thermodynamics and transport are dominated by gauge fluctuations [10]. The fluctuations of the order parameter admit a dynamical exponent $z=2$. Above the scale $E^{*}$, the fixed point exhibits marginal Fermi liquid behavior in $D=3$ (with a dynamical exponent $z=3$ ). In this intermediate energy regime the resistivity varies like $T \log T$. An important observation is that the Kondo breakdown relies on the presence of short range antiferromagnetism, which provides a small bandwidth for the f-electrons. Below a certain value of the Kondo interaction $J_{K}$, the dispersion of the f-electrons de-stabilizes the formation of the heavy metal towards a spin-liquid phase. In view of the above observation, there is no reason why, upon inclusion of magnetism into the model, the AF quantum critical point should coincide with the Kondo breakdown. The mean-field phase diagram rather suggests that the KB-QCP is generically situated under the $\mathrm{AF}$ dome (or as well under any other kind of instability, like superconductivity). Although it is not clear at the moment how the low energy regime of the KB-QCP survives the presence of nearby ordered phases, the intermediate energy regime, with linear resistivity, is expected to be a robust feature of the phase diagram.

In this Letter we address the issue of the stability of the $\mathrm{KB}-\mathrm{QCP}$ towards charge fluctuations. Our main finding is that, in a slave-boson formulation, the KB-QCP coincides with a selective Mott transition for the f-impurities. Our study is the first evidence for a selective Mott transition in the Anderson lattice. In real heavy fermion compounds, the number of f-electrons per site is not directly tunable; the valence of the impurities is allowed to fluctuate. It is commonly believed that compounds showing a large effective mass (of the order of $m^{*} \simeq 100 m_{e}$ or more) are in the heavy fermion regime where the charge of the f-impurities is frozen. It is not clear however, whether the existence of the KB-QCP is affected by valence fluctuations. To answer this question we study the Anderson lattice model (where charge is allowed to fluctuate on the f-impurities) with a small dispersion of the f-band. The situation is similar to the one encountered in the t-J 
model of cuprate superconductors at half filling $(\delta=0$ ) [14, 15], where the spin liquid phase obtained through a slave-boson formalism is believed to describe the Mott insulating state of the conduction electrons. In the Anderson lattice however, the hybridization between the impurity band and the conduction electron band is driven continuously to zero at the Mott transition, driving the system through the KB-QCP.

The possibility of a selective Mott transition in the Anderson lattice has been previously investigated in the context of single site DMFT[16]. These authors find that, at zero temperature, an infinitely small amount of hybridization destabilizes the Mott transition towards Kondo screening. At finite temperature, a first order transition terminated by a critical end-point is obtained. Our results can be reconciled with those of 16 by noticing that single site DMFT doesn't account for shortrange spin liquid effects. As such, the hybridization cannot be continuously tuned to zero within this technique. Our simple analysis suggests that when lattice effects are taken into account, the critical end-point of the Mott transition is tuned to zero for a critical value of the hybridization. We have also studied the effect of a Coulomb repulsion $U_{f c}$ between the conduction electrons and the impurity band. Within our technique $U_{f c}$ doesn't destabilize the KB-QCP.

We start with the Anderson lattice model with a small dispersion of the f-band

$$
\begin{aligned}
H & =\sum_{\langle i, j\rangle \sigma}\left(c_{i \sigma}^{\dagger} t_{i j} c_{j \sigma}+\tilde{f}_{i \sigma}^{\dagger}\left(\alpha t_{i j}+E_{0} \delta_{i j}\right) \tilde{f}_{j \sigma}\right) \\
& +\sum_{i, \sigma}\left(\left(V \tilde{f}_{i \sigma}^{\dagger} c_{i \sigma}+\text { h.c. }\right)+U \tilde{n}_{f, i}^{2}+U_{f c} \tilde{n}_{f, i} n_{c, i}\right)
\end{aligned}
$$

where $\alpha$ is a small parameter, $\sigma$ is the spin index belonging to the $\mathrm{SU}(\mathrm{N})$ representation, $t_{i j}=t$ is the hopping term taken as a constant, $V$ is the hybridization between the f- and c- bands, $E_{0}$ is the energy level of the f-electrons. $\tilde{n}_{f, i}=\sum_{\sigma} \tilde{f}_{i \sigma}^{\dagger} \tilde{f}_{i \sigma}$ and $n_{c, i}=\sum_{\sigma} \tilde{c}_{i \sigma}^{\dagger} \tilde{c}_{i \sigma}$ are the operators describing the particle number. We first study (11) in the limit of very large on site coulomb repulsion $\mathrm{U}$. In the $U \rightarrow \infty$ limit we account for the constraint of no double occupancy through a Coleman 17] boson $\tilde{f} \rightarrow f b^{\dagger}$ enslaved to a constraint on each site $\sum_{\sigma} f_{i \sigma}^{\dagger} f_{i \sigma}+b_{i}^{\dagger} b_{i}=1[18]$. Upon this transformation the effective Lagrangian writes

$$
\begin{aligned}
L & =\sum_{\langle i, j\rangle \sigma}\left(c_{i \sigma}^{\dagger}\left(\partial_{\tau} \delta_{i j}+t\right) c_{j \sigma}\right. \\
& \left.+f_{i \sigma}^{\dagger}\left(b_{i} \alpha t b_{j}^{\dagger}+\left(\partial_{\tau}+E_{0}+\lambda\right) \delta_{i j}\right) f_{j \sigma}\right) \\
& +\sum_{i} b_{i}^{\dagger}\left(\partial_{\tau}+\lambda\right) b_{i}-\lambda+\sum_{\langle i, j\rangle} J \mathbf{S}_{f i} \cdot \mathbf{S}_{f j} \\
& +\sum_{i, \sigma}\left(\left(V f_{i \sigma}^{\dagger} b_{i} c_{i \sigma}+\text { h.c. }\right)+U_{f c} n_{f, i} n_{c, i}\right)
\end{aligned}
$$

Effective hybridization and f-chemical potential

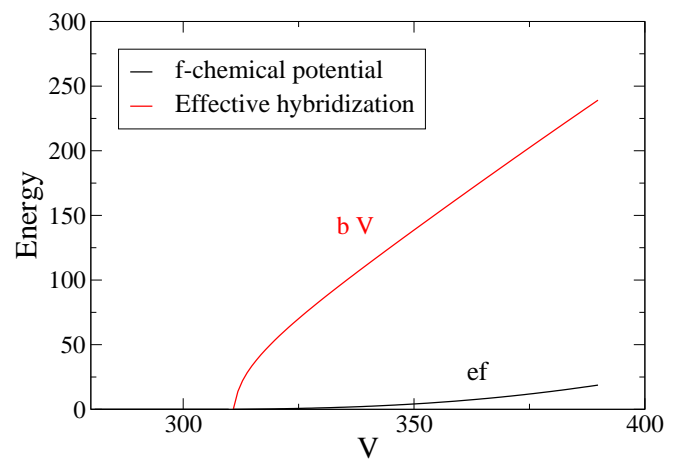

FIG. 1: Effective hybridization $V b$ and the f-band chemical potential $\epsilon_{f}=E_{0}+\lambda$ as a function of $\mathrm{V}$. The electron bandwidth is $D=1000$. The chemical potential $\mu=0$, the ratio of $\mathrm{f}$ - and c- masses is $\alpha=0.1 . \quad \beta=\left(2 \alpha^{2} t\right) / U=0.01$ and the f-energy level $E_{0}=-500$. The mean field equations are solved for $N=2$.

where $J=2(\alpha t)^{2} / U, \mathbf{S}_{i}=\sum_{\alpha \beta} f_{\alpha}^{\dagger} \sigma_{\alpha \beta} f_{\beta}$ with $\sigma$ the Pauli matrix. $\quad n_{f, i}=\sum_{\alpha} f_{\alpha}^{\dagger} f^{\alpha}$ is the density operator. The constraint has been implemented through a Lagrange multiplier $\lambda$. The term $J \mathbf{S}_{f i} \cdot \mathbf{S}_{f j}$ is generated through super-exchange mechanism, as in the t-J model for the cuprate superconductors. It is insensitive to the slave bosons. To proceed we make a static approximation where the phase of the slave bosons is frozen. The super-exchange term is decoupled in the uniform-RBV (Resonating Valence Bound) channel, which renormalizes the f-dispersion at the Hartree-Fock level, $J \mathbf{S}_{f i} \cdot \mathbf{S}_{f j} \rightarrow f_{i}^{\dagger} \beta t f_{j}$. $\beta$ is roughly constant through the phase diagram [13] and can be approximated by its value at the KB-QCP

$$
\beta=\frac{J}{t}=\frac{2 \alpha^{2} t}{U}
$$

The fc- Coulomb repulsion is decoupled using a HubbardStratonovich field $\vec{\varphi}$ such that $U_{f c} n_{f, i} n_{c, i} \rightarrow \vec{\varphi}_{i}$. $c_{i \alpha}^{\dagger} \vec{\sigma}_{\alpha \beta} f_{i \beta}+\varphi^{2} / U_{f c}$. In $\mathbf{k}$-space the mean-field equations write

$$
\begin{aligned}
& T \sum_{k, \sigma, n} b \alpha \epsilon_{k} G_{f f}\left(k, i \omega_{n}\right)+V T \sum_{k, \sigma, n} G_{f c}\left(k, i \omega_{n}\right)+b \lambda=0, \\
& T \sum_{k, \sigma, n} \vec{\sigma} G_{f c}\left(k, i \omega_{n}\right)+\vec{\varphi} / U_{f c}=0,(3 . b) \\
& T \sum_{k, \sigma, n} G_{f f}\left(k, i \omega_{n}\right)+b^{2}=N / 2,(3 . c)
\end{aligned}
$$

where $\epsilon_{k}$ is the dispersion of the c-electrons, $\epsilon_{k}^{0}=\alpha b^{2} \epsilon_{k}+$ $\beta \epsilon_{k}+E_{0}+\lambda$ is the dispersion of the f-band[20]. $G_{f f}$ and $G_{f c}$ are obtained by diagonalizing the hybridized f- and c- bands. We first set $U_{f c}=0$ leading to $\varphi=$ 0 from $\operatorname{Eqn}(3 . b)$. As depicted in figure 1, the set of mean-field equations admits a QCP where $b \rightarrow 0$ which 


\begin{tabular}{c||c|c|c}
$T \gg E^{*}$ & $C_{v}$ & $\Delta \rho(T)$ & $\chi(T)$ \\
\hline$D=3$ & $-T \log (T)$ & $-T \log (T)$ & $T^{4 / 3}$ \\
\hline$D=2$ & $T^{2 / 3}$ & $T^{2 / 3}$ & $-T \log (T)$
\end{tabular}

TABLE I: Transport and thermodynamic exponents in the Maginal Fermi liquid regime around the KB-QCP. The exponents are in agreement with those of Ref. 13].

implies that $n_{f} \rightarrow 1$ as the effective hybridization $V b$ goes to zero. At $n_{f}=1$, the impurity band is half-filled and the f-electron experience a Mott transition towards a local state. From (3.a) we notice that, at the fixed point, $V T \sum_{k, \sigma, n} G_{f c}\left(k, i \omega_{n}\right)=V^{2} \rho_{0} \log (\beta)$ with $\rho_{0}$ the density of states of the c-electrons. This leads to the standard Kondo scale

$$
\beta_{c}=\operatorname{Exp}\left[\frac{E_{0}}{N \rho_{0} V^{2}}\right],
$$

where $\mathrm{N}$ is the degeneracy of the f-electrons. In order to reach continuously the QCP it is crucial that the spin liquid parameter $\beta$ remains finite through the phase diagram.

We turn now to the fluctuations in the quantum critical regime. In a large $\mathrm{N}$ expansion, the fluctuation spectrum is in the same universality class as the KB-QCP of the Kondo-Heisenberg model[13]. We recall here the results obtained in this paper. The KB-QCP exhibits a multiscale behavior, with $z=2$ dynamical exponent below $E^{*} \simeq 0.1(q * / q)^{3} \beta D$, where $q^{*}=\left|k_{F}^{f}-k_{F}^{c}\right|$ is the difference of the Fermi level of the two species. Above $E^{*}$, the physics is dominated by dynamical exponent $z=3$ and non Fermi liquid behavior is obtained. The results are summarized in Table 1. Note that the specific heat coefficient has the same power law dependence as the corrections to resistivity. This striking property stems form the observation that in the Mott state, the f-impurities form a reservoir. The spin liquid description of the Mott state ensures (through gauge invariance) that, at the QCP, $\mathbf{v}_{f}(\mathbf{r})=0$ at each site. In a scattering process with the f-impurities, the momentum of the light conduction electrons can decay into the reservoir formed by the heavy f-fermions. Hence, although the boson propagator admits $z=3$ ( like in the proximity to a ferromagnetic QCP) the transport lifetime has no extra temperature factor compared to the electron lifetime.

In order to study the effect of $U_{f c}$ on the QCP, it is enough to keep the component of $\vec{\varphi} \| z$ in (3.b). Defining $\Pi_{f c}$ such as $T \sum_{k, n, \sigma} G_{f c}=(b V+\varphi) \Pi_{f c}$, and using the change of variables $\tilde{\varphi}=b V+\varphi$, Eqn (3.b) writes

$$
\tilde{\varphi} \Pi_{f c}+(\tilde{\varphi}-b V) / U_{f c}=0 .
$$

To answer the question of a possible first order transition in $\tilde{\varphi}$, we use (3.a) for solving for $\Pi_{f c}$, obtaining $\tilde{\varphi}_{0}=V-U_{f c} E_{0} / V$. The effective mass for the $\tilde{\varphi}$ field $m_{\tilde{\varphi}}=E_{0} /\left(V^{2}-U_{f c} E_{0}\right)+1 / U_{f c}$ is always positive, thus no first order field driven transition is present. However $U_{f c}$ shifts the QCP, leading to

$$
\beta_{c}=\operatorname{Exp}\left[\frac{E_{0}}{N \rho_{0}\left(V^{2}-U_{f c} E_{0}\right)}\right] .
$$

Eqn(16) interpolates between (44) for $U_{f c} \ll-V^{2} / E_{0}$ to $\beta=\operatorname{Exp}\left[-1 /\left(\rho_{0} U_{f c}\right)\right]$ for $U_{f c} \gg-V^{2} / E_{0}$. Note that valence transitions are known to occur in the mixed valent regime [21].

To get a deeper insight into the problem, we study the Mott transition as a function of $U$ via four KotliarRuckenstein slave bosons 22. Since no qualitative changes obtains from the inclusion of $U_{f c}$, we proceed with the model at $U_{f c}=0$. A set of four creation(annihilation) operators are introduced $e_{i}^{\dagger}\left(e_{i}\right), p_{i \sigma}^{\dagger}$ $\left(p_{i \sigma}\right), d_{i}^{\dagger}\left(d_{i}\right)$ which describe respectively zero, one or two electrons at the site " $\mathrm{i}$ ". The enlarged Hilbert space is restricted by two constraints $\sum_{\sigma} p_{i \sigma}^{\dagger} p_{i \sigma}+e_{i}^{\dagger} e_{i}+d_{i}^{\dagger} d_{i}=1$ and $f_{i \sigma}^{\dagger} f_{i \sigma}=p_{i \sigma}^{\dagger} p_{i \sigma}+d_{i}^{\dagger} d_{i}$. The Lagrangian (2) with $U_{f c}=0$ then takes the form

$$
\begin{aligned}
L & =\sum_{\langle i, j\rangle, \sigma}\left[c_{i \sigma}^{\dagger}\left(\left(\partial_{\tau}-\lambda^{(1)}\right) \delta_{i j}+t\right) c_{j \sigma}\right. \\
& \left.+f_{i \sigma}^{\dagger}\left(z_{i \sigma}^{\dagger} \alpha t z_{j \sigma}+\beta t+\left(\partial_{\tau}+E_{0}+\lambda_{\sigma}^{(2)}\right) \delta_{i j}\right) f_{j \sigma}\right] \\
& +\sum_{i}\left[e_{i}^{\dagger}\left(\partial_{\tau}+\lambda^{(1)}\right) e_{i}+d_{i}^{\dagger}\left(\partial_{\tau}+U+\lambda^{(1)}-\lambda_{\sigma}^{(2)}\right) d_{i}\right. \\
& \left.+\sum_{\sigma} p_{i \sigma}^{\dagger}\left(\partial_{\tau}+\lambda^{(1)}-\lambda_{\sigma}^{(2)}\right) p_{i \sigma}\right] \\
& +V \sum_{i \sigma}\left(f_{i \sigma}^{\dagger} z_{i \sigma} c_{i \sigma}+\text { h.c. }\right)
\end{aligned}
$$

where $z_{i \sigma}=\left(1-d_{i}^{\dagger} d_{i}-p_{i \sigma}^{\dagger} p_{i \sigma}\right)^{-1 / 2}\left(e_{i}^{\dagger} p_{i} \sigma+p_{i-\sigma}^{\dagger} d_{i}\right)(1-$ $\left.e_{i}^{\dagger} e_{i}-p_{i-\sigma}^{\dagger} p_{i-\sigma}\right)^{-1 / 2}$. The form of $z_{i \sigma}$ ensures that for $U=0$, the average $\left\langle z_{i \sigma}^{\dagger} z_{i \sigma}\right\rangle=1$. The set of mean-field equations is obtained by treating the slave -bosons in a static and uniform approximation and by differentiating the free energy with respect to $\lambda^{(1)}, \lambda_{\sigma}^{(2)}, e, p_{\sigma}, d$. The result is shown in Figure 2. First, let's fix the value of $\mathrm{U}$. At $U \geq-E_{0}$, one reaches a KB-QCP for increasing values of $V$. At low $V$ the system is in the Mott phase where the impurities are localized, while above $V=V_{c}$ a finite hybridization sets in, driving the system to a heavy metal fixed point. Alternatively fixing $V$, one obtains a line of critical points for

$$
U_{c}=\alpha^{2} t \operatorname{Exp}\left[\frac{-E_{0}}{N \rho_{0} V^{2}}\right] .
$$

For $U \leq U_{c}$ we are in the Mott phase while for $U \geq U_{c}$ we are in the heavy metal phase. The fact that Mott 


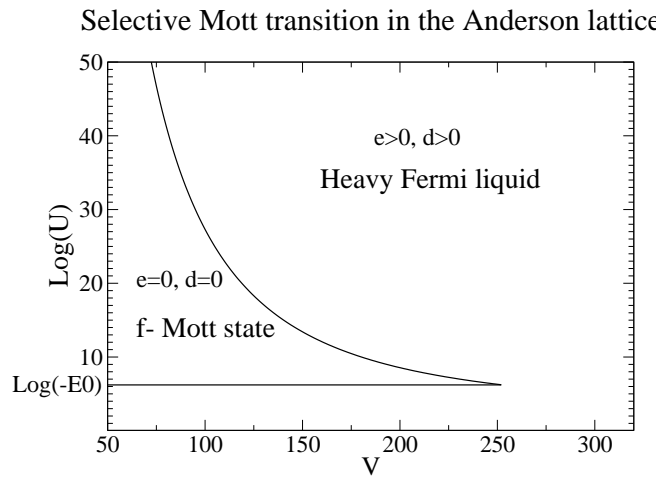

FIG. 2: Zero temperature $(\log (U), V)$-phase diagram using four Kotliar-Ruckenstein bosons. In the intermediate region the f-ipurities undergo a Mott transition. The electron bandwidth is $D=1000$. The chemical potential is taken to be $\mu=0$, the ratio of $\mathrm{f}-$ and $\mathrm{c}$ - masses is $\alpha=0.1$ and the $\mathrm{f}$ energy level $E_{0}=-500$. Here we take $N=2$.

phase breaks up at high values of $U$ follows the observation that the spin liquid parameter $J=2(\alpha T)^{2} / U$, which is necessary to stabilize the Mott phase, decreases when $U$ increases. Following [16], in the Anderson lattice with no spin liquid $(\beta=0)$, the Kondo hybridization always destabilizes the Mott phase towards a screened heavy metal. Here, the higher $V$ is, the lower is the critical $U_{c}$ at which the KB-QCP occurs. For $U \ll-E_{0}$ the Mott transition breaks down. From DMFT studies 23], the $U=-E_{0}$ line is expected to be of first order. Note also that for $V=0$ the Mott state extends to all values of $U \geq-E_{0}$, in agreement with previous studies of the half-filled Hubbard model [24].

We turn now to the discussion of our results in the light of quantum criticality in heavy fermions. In the standard scenario, AF fluctuations compete with the formation of the Kondo singlet, thus preventing the formation of the heavy metal [2, 8]. Although it has been shown that the one impurity Kondo screening is inhibited by AF fluctuations [25, 26], in the Kondo lattice, however, the question of whether AF fluctuations are strong enough to destroy the heavy Fermi liquid remains open. If the standard scenario is correct, the Kondo breakdown should occur at the point in the phase diagram where AF fluctuations are maximum, namely at the AF QCP. Alternatively our study suggests that the Kondo breakdown occurs at the point where the f-impurities are subject to a selective Mott transition. Within our study, a small non vanishing dispersion of the spinon band is the necessary and sufficient condition for the existence of the KB-QCP. The second scenario thus relies on the presence of a spin liquid component of the short $\mathrm{AF}$ fluctuations at the Mott transition. The question of the validity of the spin liquid description of the Mott transition dates from the early days of high $T_{c}$ superconductivity with the idea of Resonance Valence Bond (RVB) around half filling in the Hubbard model[14]. Studies of frustrated magnetism have concluded that, in the absence of charge fluctuations, spin liquid phases can be induced by frustration 27]. However, no mixed phase consisting of $\mathrm{AF}$ and spin liquid has been found. In the presence of charge fluctuations, like around zero doped cuprate superconductors, it is still unclear whether a short range RVB state exists or not 24]. Our study of the Anderson lattice provides us with a situation where charge fluctuations are strong (through coupling to the conduction band), rendering the occurrence of the spin liquid more favorable. The presence of the selective Mott transition in this model is thus a direct test for the existence of a short range RVB spin liquid, stabilized by charge fluctuations.

We thank the hospitality of the KITP(Santa Barbara) for the "Quantum Phase Transition (2005)" workshop where this work was initiated. Useful discussions with G. Kotliar, M. Norman, O. Parcollet, I. Paul and J. Rech are acknowledged.

[1] G. Stewart, Rev. Mod. Phys. 56, 755 (1984); 73, 797 (2001).

[2] P. Coleman et al., J. Phys. Cond. Matter 13 R723 (2001).

[3] H. v. Löneysen et al. cond-mat/0606317.

[4] J. A. Hertz, Phys. Rev. B 14, 1165 (1976).

[5] A. J. Millis, Phys. Rev. B 48, 7183 (1993).

[6] T. Moriya and T. Takimoto, J. Phys. Soc. Japan 64, 960 (1995).

[7] A. Rosch et al., Phys. Rev. Lett. 79, 159 (1997); A. Rosch ibid 82, 4280 (1999).

[8] Q. Si et al. Nature 413, 804 (2001); D.R. Grempel and Q. Si, Phys. Rev. Lett. 91, 026401 (2003).

[9] P. Sun and G. Kotliar, Phys. Rev. Lett. 91, 037209 (2003).

[10] T. Senthil et al., Phys. Rev. Lett. 90, 216403 (2003); Phys. Rev. B 69, 035111 (2004).

[11] S. Doniach, Physica B, 91, 213 (1977).

[12] P. Coleman, J. B. Marston, A. J. Schofield, Phys. Rev. B 72, 245111 (2005).

[13] I. Paul, C. Pépin and M. Norman, Phys. Rev. Lett. 98, 026402 (2007).

[14] P.W. Anderson, Science 235, 1196 (1987).

[15] J. Marston and I Affleck, Phys. Rev. B 39, 11538 (1989).

[16] L. de' Medici et al . Phys. Rev. Lett. 95, 066402 (2005).

[17] P. Coleman, Phys. Rev. B 29, 3035 (1984).

[18] Note that the technique with one slave boson treats the low energy part of the model, with no information about the Hubbard bands.

[19] N. Read and D. M. Newns, J. Phys. C 16, 3273 (1983); N. Read, J. Phys. C 18, 2651 (1985).

[20] We are considering the uniform condensation of the slave bosons, occuring when the masses of c- and f-bands are of the same sign [13]. When masses are of opposite sign the condensation occurs at finite $q$.

[21] A. T. Holmes et al. Phys. Rev. B 69, 024508 (2004); Y. Onishi and K. Miyake J. Phys. Soc. Japan 69, 3955 (2000).

[22] Phys. Rev. Lett. 57, 1362 (1986). 
[23] A. Georges et al. Rev. Mod. Phys.68 13-123 (1996).

[24] see P. A. Lee, N. Nagaosa and X-G. Wen, Rev. Mod. Phys. 78, 17-85 (2006) and Ref. therein.

[25] A. Larkin and M. Mel'nikov, Sov. Phys. JETP 34, 656 (1972).
[26] H. Maebashi, K. Miyake and C . Varma, Phys.Rev.Lett. 95,207207 (2005).

[27] R. Moessner and S. L Sondhi, Phys. Rev. Lett. 86, 1881 (2001). 